\title{
Rules as Basic Units of Sociocultural Selection
}

\author{
Julio C. Aguiar ${ }^{1}$ - Jorge M. Oliveira-Castro ${ }^{2,3}$ (I) $\cdot$ Leandro Gobbo $^{4}$
}

Published online: 28 May 2019

(C) Association for Behavior Analysis International 2019, corrected publication 2019

\begin{abstract}
In the context of applying behavior-analytic principles to the study of the evolution of human societies, we propose that rules constitute the basic units of sociocultural selection. Such thesis is based on a critical analysis of the ideas concerning sociocultural selection proposed by Skinner and Baum. Its point of departure is a new functional concept of rule uttering, defined as a special type of verbal behavioral pattern, whose probability of occurrence depends upon their probability of altering the frequency of certain kind of behavior in the repertoire of a given individual or group of individuals. These relatively lasting changes in the behavior of recipients include the uttering of rules appropriate to specific types of problem situations, which increase the likelihood of responses that solve the problems faced by the social group. According to the proposed model, rule-uttering behavioral patterns include rule application, rule transmission and rule creation, which are functionally distinguished by the social consequences they produce. Patterns of rule uttering are selected by operant mechanisms, in the sense that those that do not increase the probability of solving problems tend to stop occurring. It is proposed that the occurrence probability of generalized social reinforcers, associated to different functionally specialized social systems (e.g., money in the economy, sanctions in the legal system), constitutes the main consequence responsible for rule-uttering selection.
\end{abstract}

Keywords Behavior analysis - Sociocultural selection - Rule uttering · Selection of rules · Generalized social reinforcers $\cdot$ Functionally specialized $\cdot$ Social systems

Jorge M. Oliveira-Castro

jorge.oliveiracastro@gmail.com; jocastro@unb.br

1 Catholic University of Brasilia, Brasilia, Brazil

2 University of Brasilia, Brasilia, Brazil

3 Instituto de Psicologia, Universidade de Brasilia, Campus Universitário Darcy Ribeiro, ICC-Sul, Brasilia, DF 70910-900, Brazil

4 Pioneer Union of Social Integration, Brasilia, Brazil 
Starting with Skinner, the application of behavioral analysis to the study of the evolution of human societies has been marked by two basic assumptions. The first one states that such a study necessarily involves a third level of selection by consequences, namely the sociocultural level; the second one states that the basic units of selection at the sociocultural level are the so-called cultural practices.

Such common basis, however, does not preclude the existence of several distinct and not entirely convergent research programs within the radical behaviorist approach to human sociocultural selection. Among them, and beyond the seminal texts of Skinner (1953, 1981, 1988), we highlight the research program led by Glenn $(1988,2003,2004)$, centered on the concept of metacontingencies, and the studies of Baum (1995, 2000, 2005), more focused on the concept of cultural practices and convergent with the adepts of sociobiology and the so-called genetic-cultural coevolution - both paramount due to their repercussion in the behavior-analytic field. Furthermore, we can mention the studies of Kunkel (1975, 1986, 1991) on social behavior, rules, and social structure, among other relevant studies.

In order to contribute to this debate, in a theoretical level, this article addresses the question of what the basic unit of sociocultural selection is, based on a critical analysis of the ideas on this subject advanced mainly by Baum and Skinner. The structure of the article is as follows: The first section discusses the concepts of social behavior, social contingencies, and cultural practices. The second proposes a functional concept of rules (rule uttering). The third is dedicated to explaining in detail the thesis that rules are the basic units of sociocultural selection. The fourth section proposes a rule-selection model. The final section summarizes the main points of the article.

\section{Social Behavior, Social Contingencies, and Cultural Practices}

The starting point for any discussion on sociocultural selection from a behavior-analytic perspective is the concept of human social behavior. According to radical behaviorism, the concept of human social behavior does not concern the behavior of collective entities, such as tribes or nations, but refers instead to the subset comprised of the behavioral repertoire of an individual whose controlling contingencies are composed of behavioral patterns of other individuals (Baum, 2005). When they form a recurrent functional unit, the interlocked behavioral patterns of distinct individuals form what Skinner called social systems, each instance constituting a social episode (Skinner, 1953, 1957). Considered in relation to each other, the behavioral patterns that make up a social system function as reciprocal behavior-controlling contingencies, named social contingencies by Baum (2000, p. 188).

As we will stress throughout this article, due to the social nature of our species the acquisition, maintenance, or extinction of human individual behavior is always a consequence, to some extent, of social contingencies, which are formed by the behavior of other people as a response to our behavior in the various contexts that make up a human life in any time or place. The importance of the reaction of others to our behavior renders laboratory reproduction or even the formal modeling of human behavioral patterns extremely challenging, amid other reasons, due to the influence of the quantity of people considered relevant to the determination of behavior (Simmel, 1902).

Skinner attempted to solve part of this problem by distinguishing between (1) social situations in which someone's behavior is reinforced by an environmental change that 
depends on the mediation of someone else's behavior, from (2) social situations in which an individual is merely the object of another individual's behavior (Skinner, 1953, pp. 298-299). Such a differentiation, however, though conceptually possible, is theoretically and empirically futile. Skinner's own examples prove it. For instance, in the case of two boxers, it is possible to argue that, even assuming that, from the point of view of each fighter, the opponent is only the object of their respective fighting behaviors, the most relevant point is that the social reinforcement for the boxers' behavior does not come from each fighter in relation to the other. It is also provided directly by reactions from the public, and indirectly from the sponsors of the fight, associations of boxers, and so on. Therefore, it is better to define the concept of social contingencies as the set of human behaviors that directly or indirectly exert influence in the acquisition, maintenance, or extinction of other human behaviors. Of course, such a generic concept assists relatively little in the specific task of building a model of human sociocultural selection, which brings us to the concept of cultural practice.

Unlike the concept of operant, which can be defined in strictly operational terms (i.e., based on observations from rigorous laboratory experiments [Skinner, 1953, 1989; Catania, 1998]), the concept of cultural practice is purely theoretical and as such its meaning depends on the broader theoretical context of which it is part. Thus, and it is not a surprise, there are several more or less explicit definitions of such concept, which vary according to the several research programs on sociocultural selection extant in the behavior-analytic field.

For the purposes of the present article, a definition that can serve as a starting point is that of Baum, who defines cultural practices as "all behavior patterns acquired as a result of membership in a group" (Baum, 2000, p. 187). Nonetheless, given what we have said about the social nature of virtually all human behavior, it is important to consider Skinner's remark concerning the various meanings of the expression "cultural practice," to emphasize that, in the context of a theoretical definition, such an expression must refer to a kind of behavioral pattern. It is also advisable, following Skinner's teachings, to replace the subjective notion of "membership in a group" with a more objective one, such as "characteristic of a particular group." Then, we can define cultural practice as a kind of behavioral pattern characteristic of a particular group of human individuals (Skinner, 1989, pp. 86-87).

It should be noted that, although behavioral patterns are always individual, in the sense that, as Skinner (1953, p. 311) says, "[i]t is always an individual who behaves," the contingencies responsible for the emergence, transmission, and reproduction of a kind of behavioral pattern in a given group of individuals are not equivalent to those responsible for the acquisition and maintenance of the respective individual behavioral patterns. That is, ontogenetic and sociocultural behavioral selections are orthogonal to each other. As Skinner says, "[i]t is the effect on the group, not the reinforcing consequences for individual members, which is responsible for the evolution of the culture" (Skinner, 1981, p. 502).

Indeed, even in the case of new cultural practices, whose emergence we may legitimately attribute to variations in the behavioral repertoire of a particular individual, when observed from a historical perspective those practices are better described at the time of their emergence as individual variations arising within preexisting kinds of behavioral patterns characteristic of the culture at the time. In the case of the cultural practice associated with Freudian psychoanalysis, for example, whose emergence can be attributed to variations in 
the repertoire of Austrian neurologist Sigmund Freud, the variations in his repertoire arose in the context of preexisting behavioral patterns in psychiatry in general or, in particular, the treatment of so-called neuroses (Mezan, 1982). On the other hand, the very fact of the successful reproduction of the new behavioral pattern indicates a concomitant propensity of other individuals in the group to reinforce and reproduce such behavior, which cannot be explained exclusively by the particular contingencies of each individual biography. For example, if someone is a professional boxer, the behavior analyst will seek in the behavioral history of that individual the contingencies responsible for the acquisition and maintenance of the behavioral repertoire known as professional pugilism. Nevertheless, it is in the history of the culture in which the individual in question lives that the behavior analyst must investigate the social contingencies responsible for the emergence and reproduction of this cultural practice, particularly the behaviors of those who pay for the fights - spectators and sponsors - without which it would not be possible to professionalize the boxers.

Still, considering the question of what the basic unit of sociocultural selection would be, the concept of cultural practice proposed above is not the answer, because, for the most part, the relevant contingencies in the context of sociocultural selection are those present at a group level and not those that govern the behavior of individuals considered by themselves, including, within the latter, social contingencies. Not every cultural practice - that is, not every behavior pattern characteristic of a social group - influences the probability of group reproduction and survival. Thus, it is necessary to pinpoint what specific kind of cultural practice one has in mind when he or she speaks of sociocultural selection. The answer lies in the concept of rules.

\section{A Functional Concept of Rules}

The role of rules in the selection of cultural practices is mainly discussed in Baum's theoretical studies on this subject (Baum, 1995, 2000). Notwithstanding, as this author points out, the theorization of cultural practices among behavior analysts has not emphasized this aspect and therefore has not given the concept of rules its deserved prominence in the development of the radical behaviorist approach to sociocultural selection (Baum, 1995, p. 3). In our view, a crucial step in this direction would be to shift the focus of the research on rules from their form (topography) to the contingencies responsible for their emergence, transmission, modification, or extinction. In other words, we need to replace the merely topographic concept of rules as "contingencyspecifying stimuli" proposed by Skinner (1969, pp. 146-149) and accepted by most behavior analysts for a more functional one.

Both Skinner and Baum suggest approaches for a functional definition of rules. Skinner $(1969,1989)$ emphasizes the context in which rules occur more frequentlythat of problem solving. Baum (2000, p. 194) asserts the role of rules in the selection of behavioral patterns among the members of a given culture. Neither of them, however, came up with a purely functional definition of rules, which would explain its idealtypical, contingent-specifying form or topography, instead of taking that form as the basis of the definition itself. In Baum's case, such a failure is purportedly corrected by the postulation of an inherently beneficial character of rules, through the thesis that they always involve two contingencies of reinforcement (or reinforcement relations, as he prefers): a proximate one, related to social reinforcement for following the rule; and an 
ultimate one, resulting from the long-term adaptive benefit provided by the rule, both for the individual who follows it and for its formulator or utterer (Baum, 2005, pp. 166171). Among other shortcomings, such a postulate seems to be unjustified in historical terms, given the relative abundance of examples, emphasized by various authors, of cultures whose legal rules are beneficial only to part of their members to the detriment of others (Alexander, 1987; Betzig, 1986; North, Wallis, \& Weingast, 2009; Acemoglu \& Robinson, 2012). In fact, Baum's thesis on ultimate or distant contingency is, as we shall argue below, relevant to the understanding of social rules' selection mechanism. However, as part of a definition of rules, it is incorrect, because not all rules are directed to a whole society, and even those that are might not be beneficial to all its members but only to a powerful group within society. What then would be an adequate, functional definition of rules?

Based on what has been said, we propose to define rules as a special kind of verbal behavioral pattern whose probability of occurrence depends on its ability to change the frequency of occurrence of a certain type of behavioral pattern in the repertoire of a given individual or group of individuals. This definition has three distinctive features, presented below, which we think are important from the point of view of a theory of sociocultural selection.

First, the problems associated with the notion of verbal stimuli as a "product of an individual's verbal behavior" (Michael, 1982, p. 2; Schlinger, 1993, p. 9), implicit in the classic definition proposed by Skinner, are avoided. The verbal or nonverbal nature of a stimulus is determined not only by the efficient cause of such stimulus, but by the functional relationship between it and the behavior that follows it (Luhmann, 1990; Corsi, Esposito, \& Baraldi, 1996; Moeller, 2006). For example, a fragment of the Hammurabi Code, written in Akkadian, although being a "product of an individual's verbal behavior," cannot be considered a "verbal stimulus" for most of the billions of people who inhabit the Earth today. Likewise, the peculiar sounds (i.e., clicks) present in the language of the Khoikhoi people of South Africa, for those who have never seen the social context in which such sounds are emitted, could easily be taken as nonverbal. In fact, the concept of verbal behavior as a kind of social behavior, distinguishable from others only by its peculiar topography, cannot be understood by considering only one of its component behavioral patterns, be it the speaker's or the listener's behavior-or for that matter the writer's or reader's. This is so because verbal behavior is a social relation or, as Skinner argues, a type of social system (recurrent social event), by definition involving at least a pair of interlocked behavioral patterns, as explained above (Skinner, 1957, 1989).

Directly linked to the previous point, we have the second distinguishing characteristic of the proposed definition of rules, which is the emphasis on the contingency of reinforcement existing between the behavior of the person who utters the rule and the person to whom it is addressed, respectively. In other words, the rules - as highlighted by Skinner when discussing legal rules (Skinner, 1953, pp. 333-349) —are types of verbal behavior whose frequency of occurrence is determined by the degree of control exercised over the behavior of other (heterocontrol) or the same person (self-control).

Notwithstanding their critical importance, these are not the most important distinctive properties of rule uttering. The third and most significant distinguishing characteristic of the proposed definition of rule uttering is the fact that the control exercised by them is not merely episodic or circumstantial, but a relatively lasting change in the behavioral repertoire of their recipients. Thus, a command of the type "give me your 
money or die" is not usually an instance of rule uttering, because the reinforcement for such behavior is not the change of the behavioral repertoire of the recipient of the command but simply the delivery of money. Likewise, an instruction such as "fix the table part with the round head metal screws" found in a table assembly manual is not a case of rule uttering, because it is solely reinforced by modeling the behavior of mounting the table. In these two cases there is no relatively lasting change in the behavioral repertoire of their recipients.

Conversely, a statement such as the Law of Universal Gravitation- "a particle attracts every other particle in the universe using a force that is directly proportional to the product of their masses and inversely proportional to the square of the distance between their centers" - is an example of a rule. It counts as an example of a rule because its frequency of occurrence is dependent on the reinforcement represented by the relatively lasting change in behavior (verbal and nonverbal) of its potential recipients. ${ }^{1}$ A legal norm such as the prohibition of theft within Brazilian Criminal Law"Article 155: Unlawfully taking, for themselves or others, personal property. Penalty: imprisonment, from one to four years, and a fine" - is also an example of a rule. It is so because the frequency of uttering it depends on the reinforcement provided by the change in the behavior of judges, lawyers, and prosecutors - in short, all those who have to deal with the problem of penalizing (or not) the conduct described in the rule.

Thus, according to the concept of rules mostly adopted in behavior-analytic literature (e.g., Skinner, 1969, 1989), all four examples cited above could count as instances of rules and rule-governed behavior. In all cases, considering the behavior of the recipient (listener, reader), there is verbal behavior whose topography specifies a contingency between certain responses and consequences, which, for verbally trained audiences, functions as discriminative context that indicates an increased probability of reinforcement (or punishment) for certain responses. On the other side of the interaction, when one analyzes the behavior of the speaker (or writer), in all cases the uttering response would be reinforced if the recipient emits behavior compatible with the topography associated to the verbal utterance. However, in the last two examples, there are additional elements in the consequences that reinforce uttering responses: the uttering behavior would be reinforced by lasting changes in the behavior of the recipients, that is, modifications in their repertoires, including the behavioral pattern of uttering the rule in problem situations (e.g., an engineer making calculations, a judge writing a ruling) and behaving according to the rule. The first two cases illustrate what some have called rule following (e.g., Baum, 2000, 2005), whereas the last two cases exemplify instances of what we propose to call rule application. As will be argued below, the characteristic of rule-uttering behavior of having their frequency of occurrence dependent on a greater or lesser change in the behavioral repertoire of the respective recipient is crucial to understanding of their role in sociocultural selection. Yet it is virtually absent from discussions about this subject among behavior analysts.

We suspect that this is due, in large part, to the fact that the focus of such discussions has hitherto been on the question: What are the basic behavioral mechanisms by which rules, defined as contingency-specifying stimuli, control the behavior of their recipients (Malott, 1989, p. 289)? This question is meaningful and relevant. Even so, it cannot be

\footnotetext{
${ }^{1}$ As explained by Skinner (1969), "scientific laws also specify or imply responses and their consequences. They are not, of course, obeyed by nature but by men who deal effectively with nature" (p. 141).
} 
confused with a different question concerning the contingencies responsible for the behavior of the utterers and recipients of the rules as a social phenomenon. This is because, although the first question concerns the basic behavioral mechanisms, being therefore a topic pertaining to the analysis of human operant behavior in general, the second question pertains to a different level of analysis, namely social behavior, in the sense that it involves the reciprocal operant control between the interlocked behaviors of the utterer and the recipient of the rules as constitutive aspects of social systems, i.e., recurrent social events. It is intuitive that, whatever the explanation for the first type of question, the second will remain unanswered if it does not receive a theoretical and empirical treatment of its own within behavior analysis.

Given that the research has so far focused on basic behavioral aspects, the concept of rules has been defined too broadly, including different types of verbal behavioral patterns (Skinner, 1989; Zettle, 1990). From a sociocultural selection theory standpoint, however, it makes sense to conceptually differentiate those behavioral patterns that are reinforced by the ability to change the recipient's behavioral repertoire from those reinforced by other types of behavioral change. For example, someone who makes the promise "I'll never drive drunk again" will be under control of reinforcement contingencies of different kind from someone who says "I'll meet you at the club in 30 minutes." For the same reason, a proposition of the type "When solving a problem, ask yourself: what is the unknown?" is distinct, in terms of reinforcing contingencies, from "The exercise must be delivered until next Friday." In cases involving the promise and the problem-solving heuristic, it is clearly a matter of changing the recipient's behavioral repertoire (who may be the utterer of the rule her-/himself) whereas in the other cases, reinforcement of the verbal statements is obtaining control over the recipient's behavior, but only in a circumstantial and momentary way. It is possible that in terms of basic processes it is not relevant to distinguish between them. In social terms (i.e., in terms of the reciprocal contingencies between utterer and recipient), however, these are clearly different contingencies. For the purposes of the behavior-analytic theory of sociocultural selection, it is the social rather than the individual behavioral contingencies that truly matters in the conceptualization of rules. That being said, there remains the question: to what extent is the proposed functional definition compatible with the proposition of considering rules as the basic units of sociocultural selection?

\section{Rules as the Basic Unit of Sociocultural Selection}

Among behavior analysts, Baum (2005) is the only one who nearly arrives at the proposition of the rule as the basic unit of sociocultural selection, especially when he distinguishes between imitation-based cultures and those based on instruction. He even discusses extensively the selection of rules (Baum, 2000). Still, because he remains attached to the topographical concept of rules as contingency-specifying stimuli, he ends up seeing them only as contexts in which cultural practices occur (Baum, 2000, p. 209). Thus, the role of basic units of sociocultural selection is awarded to cultural practices in general, without the above-mentioned distinctions.

The major problem with the status of cultural practices in general-that is, any type of behavioral pattern characteristic of a given culture - as the unit of sociocultural selection is that the very concept of sociocultural evolution is lost, becoming synonymous with 
cultural drift. Although the concept of cultural drift is not unanimous in the literature, more representative versions stress the random changes in cultural practices as its common feature (Eggan, 1963), which, as will be argued below, do not account for the kind of directional (i.e., teleonomic) changes that characterize sociocultural evolution (Skinner, 1971; Corning, 2014). This is probably one of the reasons why Baum-following other authors in the gene-culture coevolution paradigm - eventually subsumes sociocultural evolution into biological evolution by directly linking the selection of cultural practices to their contribution to biological fitness (Baum, 2005, p. 283).

Skinner, in this regard, embraces a more promising stance by saying that "[a] culture evolves when practices originating in this way [i.e., through individual discoveries on better ways of performing typical tasks of human life] contribute to the success of the practicing group in solving its problems" (Skinner, 1981, p. 502). Two points in this passage are particularly relevant to our discussion. First, the allusion to better ways of doing things entails the proposition that the concept of sociocultural evolution implies the idea of directional changes, which Campbell (1974), based on Popper, has called "teleological achievements." Skinner (1971, p. 143) goes so far as to suggest, in another passage, that the common direction to the processes of biological and sociocultural evolution is to make organisms increasingly sensitive to the consequences of their actions. This point is fundamental, because it excludes mere sociocultural drift from the study of the evolution of human cultures. In other words, accepting Skinner's conjecture, only those evolutionary achievements, to use Luhmann's (1985) expression, which contribute to a greater sensitivity to the consequences of human actions are considered links in the process of sociocultural evolution. Second, the hypothesis that consequences in terms of success in solving the problems of a group, and not the individuals considered separately, determine the selection of cultural practices. Thus, the question that arises is: in what way does culture work to solve a group's problems?

As in Baum, Skinner's (1969) response clearly points to the effect of rules in modifying the behavioral repertoire of individuals belonging to a given culture, when he explains that "[a]s a culture produces maxims, laws, grammar, and science, its members find it easier to behave effectively without direct or prolonged contact with the contingencies of reinforcement thus formulated" (p. 141). He, however, does not persevere in this line of thought and, soon after retakes the position that rules are "discriminative contingency-specifying stimuli." Whether this definition is accurate is debatable (Blakely \& Schlinger, 1987; Malott, 1989; Schlinger, 1993). In any case, from the perspective of research on the role of rules in sociocultural selection, this question is peripheral, if not wholly irrelevant. What is important is that rules allow individuals to change the behavioral repertoire of other individuals without it being necessary to make them experience the respective contingencies directly, only the social contingencies arising from following or not following the rules. As Malott (1989) mentions, this is particularly the case with respect to what he calls "contingencies that are not direct acting," such as those involving consequences that are too distant in time, highly unlikely, or dependent on the accumulation of consequences to influence behavior. It should be noted that many of the problems that affect the group are framed by this type of contingency (Hardin, 1968; Boudon, 1977; Schelling, 1978; Gosselin, 1998).

In the behavior-analytic field, the researcher who has most studied the equivalence between the ineffectiveness of operant behavioral control by consequences that are too remote in time and those that greatly affect the group as a whole, but only marginally the 
individuals who comprise it, is Rachlin (2000, pp. 165-193). In his studies, he underlines the role of rules in maintaining behavioral patterns whose reinforcement is distant in time or dependent on coordinated cooperation between different individuals (pp. 142143). Nevertheless, Rachlin barely discusses the question of sociocultural selection, and therefore fails to link his thesis on the equivalence between self-control and social cooperation to the question concerning the function of rules in the evolution of cultures.

In our opinion, in addition to the absolute predominance of discussions about the basic behavioral processes related to following rules in the behavior-analytic field, which ends up strengthening the topographic definition of rules as contingency-specifying stimuli, a further decisive factor for the underdevelopment of the discussion about the role of rules in sociocultural selection is most behavior analysts' uncritical acceptance of methodological individualism (Moore, 2008). Such acceptance contributes decisively to the fact that Skinner's thesis - according to which collective effects, rather than individual reinforcement contingencies, are responsible for the selection of cultural practices - has not been properly considered. Even Baum, who discusses Skinner's notion of cultural practices as synonymous with social contingencies, does not formulate a functional concept of rules that might explain typical cases of contingencies that "do not act directly" (Malott, 1989). Baum's functional concept does not connect the issue of contingencies of survival and reproduction of the group as a whole (Skinner, 1971, 1976, 1981, 1989) to the function of rules as a means of changing the behavioral repertoire of individuals without the need of exposing them to such contingencies.

Summarizing the discussion so far, we can state that the proposed functional definition of rule uttering allows us to connect rules directly to the solution of the problem of how human groups adapt to contingencies at the group level (which might be termed sociocultural contingencies), which are of the type referred to, by Malott, as contingencies that "do not act directly." According to the present proposal, rule uttering is defined as a special kind of verbal behavioral pattern whose probability of occurrence depends on its ability to change the frequency of occurrence of a certain type of behavior pattern in the repertoire of an individual or group of individuals. That said, there remains a central question to be answered, so that we can define the rules as the basic unit of sociocultural selection, namely: how is it possible that rules get to be formulated and, in particular, selected, given that the sociocultural contingencies do not act directly on behavior?

Skinner's and Baum's solutions to this question, as discussed, are unsatisfactory for three reasons. First, they are not directly related to the premise of sociocultural selection as a selection of rules, in the abovementioned functional sense. Second, they postulate an identity of interests between the utterer and the recipient of rules, which is not in line with the facts of social life in many cultures. Third, and most important, they do not clearly distinguish between individual contingencies - which include the immediate and directly acting, social and nonsocial — and sociocultural contingencies, that is, those that exist in a level beyond the individual, although not necessarily related to the group as a whole (Skinner, 1981; Baum, 2005).

Baum's $(1995,2000,2005)$ solution basically consists of relating what we are calling sociocultural contingencies with the biological concept of fitness. Skinner (1988), on the other hand, although he often mentions the contingencies of survival and reproduction of the group, fails to propose a minimally developed model of sociocultural evolution based on such contingencies, merely reaffirming that, beyond 
its effects at the individual level, cultural mutations usually have other effects "which can be stated only at the level of the group in spite of the fact that it is always an individual who behaves" (p. 47).

In the next section, we will propose a rule-selection model based on a typology of rule-uttering behavioral patterns and the functional specialization of social systems made possible by the emergence of second-degree rules.

\section{The Rule-Selection Model}

A rule-selection model must include, on the one hand, the kinds of behavioral patterns that comprise what we have called rule-uttering behavior. On the other hand, it also must include the mechanism by which rules are selected to become part of a given culture and remain or not as such. Let us first look at the types of behavioral patterns that make up rule-uttering behavior.

\section{A Typology of Rule-Uttering Behaviors}

In his study of the role of rules in sociocultural evolution, Baum $(2000,2005)$ distinguishes three types of behavioral patterns that fit into what we call rule uttering. They are rule making, rule giving, and rule following. Baum (2000) also directly associates the occurrence of such behavioral patterns with natural selection, using examples ranging from brushing teeth to eating taboos, such as the prohibition of eating pork among the precepts of Judaism (p. 202). From the perspective of elaborating a theoretical model of rule selection, such classification, although an acceptable starting point, needs to be refined in some important respects.

In our view, the first and main problem concerns the rule-following pattern, which has been usually interpreted as the observed correlation between presentations of a contingency-specifying verbal stimulus and emissions of a response compatible with the topography related to the stimulus. This would be the case of behavior under the control of commands like the written word "STOP" of a traffic sign, as when someone stops the car every time they are faced with such sign. In this example, because there are sources of reinforcement for the response of driving through the sign without stopping (i.e., shorter travelling time), it can be asserted that the stopping response pattern is under the control of the road sign and the behavioral history of the individual. The prevailing relations between the rule and responses in this type of behavioral pattern differs functionally from relations between the rule and responses in the case of a student solving arithmetic problems or of a judge sentencing criminal cases. They are not following rules, in the sense of the traffic sign example, in which the presence of the sign functions as discriminative stimulus for stopping. The student and the judge face problem situations that require the emission of precurrent responses, which consist of rule utterances that increase the likelihood of final responses that might solve the problem (cf. Skinner, 1969). These precurrent responses may include looking up support materials, such as texts, third opinions, and videos, which increase the likelihood of adequate rule uttering and the emission of responses that might be reinforced by solving the problem. When compared to the rule-following example involving the traffic sign, in these latter cases there is no discriminative stimulus available in the 
initial setting, such as the "STOP" sign, that controls the emission of the correct response. The problem situation contains events that function as discriminative stimuli to rule uttering, which, in turn, produce discriminative stimuli associated to a correct response. Rule uttering generates events, such as the statement " $a \times(b+c)=a \times b+a$ $\times c$," which increases the probability of correct responses to the problem, in a manner similar to the function played by the traffic sign. It is possible to consider that in the traffic sign case, when the person was beginning to learn how to drive, there might also have been precurrent responses of uttering rules, which, as training increased, stopped occurring (cf. Oliveira-Castro, Coelho, \& Oliveira-Castro, 1999; Oliveira-Castro, Faria, Dias, \& Coelho, 2002). But the concept of rule following, as it has been adopted in the field, does not consider such functional distinctions. Later in this section, we will propose replacing the concept of rule following with that of "rule application," which refers to the precurrent behavior of uttering rules in special contexts called problem situations.

With respect to the rule-giving pattern, the problem is the assumption, implicit in the term, of an altruistic motivation on the part of the rule giver in relation to the recipient. Simply think of a rule such as "If you approach my daughter again, I'll break you," to realize that the aforementioned assumption is false. Thus, it is much more plausible to postulate, as in the case of this article, that rule-uttering behavior has, as its reinforcing variable, the change in the behavioral repertoire of the recipient, without any assumption concerning beneficial effects that may result from this change. At any rate, this concept fulfills a function in Baum's rule-selection model that we believe would be better fulfilled by the concept of "rule transmission," which describes the process by which a rule is passed from an individual to another-usually but not necessarilythrough teaching (Skinner, 1968; Baum, 2000). Thus, rule transmission is not an individual behavioral pattern but a social system involving at least two behavioral patterns, namely, to convey and to adopt the rule, respectively. Such a social system is characterized by the fact that both the one who conveys and the one who adopts the rule are reinforced by the behavioral change in the latter, which consists, not only or mainly of learning the behavior described in the rule, but learning the rule itself. In other words, the behavior of transmitting a rule, emitted by the person who utters the rule, is reinforced by the behavior of applying the rule by the recipient.

Regarding the rule-making pattern, the caveat we make is on three points of Baum's explanation. First, the idea that what the rule maker observes are predominantly regularities in the natural environment and not human behavioral patterns, as in the example of caries decay (Baum, 2000, 2005). It is more plausible that some practice functionally like tooth brushing has arisen and been reported or transmitted verbally, giving rise, much later, to the rule that we should brush our teeth. Second, and directly linked to the first point, a rule-making pattern, as a distinct cultural practice, must have arisen later than the rule-giving pattern (which we propose to replace with "ruletransmission pattern," for reasons stated above), as suggested by Skinner (1988). In fact, the rule-making pattern presupposes the existence of a cultural practice of transmitting rules, so it must have evolved later. Third, the rule-making pattern (in our terminology, "rule creation"), in view of the functional definition of rule proposed (which emphasizes the change in the recipient's repertoire as a reinforcer of any rule uttering, including its creation), does not need to produce the fitness of the recipient as a mediated or immediate reinforcer. What matters for its recurrence is the ability to 
change the behavior of the addressee or addressees, particularly by introducing the rule in question in the respective behavioral repertoire.

Based on this critical analysis of Baum's terminology, we propose the following typology of rule-uttering behaviors: "rule application," "rule transmission," and "rule creation." Let us take a glimpse at each one.

Rule application occurs in the context of a problem, i.e., a situation in which "we cannot emit a response which, because of some current state of deprivation or aversive stimulation, is strong" (Skinner, 1968, p. 132). It is important to note that a rule being part of the behavioral repertoire of an individual or group of individuals does not exclude situations in which it is necessary to consult a text in order to utter the rule. For example, an experienced cellist may consult a musical score whenever he or she performs certain music, which obviously does not mean that the score in question (that is, the rule) is not part of his repertoire. It occurs that many of the behaviors that are part of the repertoire of individuals in our hypercomplex society depend, if they are to be emitted, on the aid of texts and other contextual elements apt to evoke such behaviors. The behavioral repertoire of the judge who, whenever he or she is going to issue a ruling for any kind of case, consults the relevant laws, doctrine, and precedents, certainly includes the legal rule applicable to the case, which does not preclude consultation of the mentioned texts, as way to strengthen that behavior (Skinner, 1953). Such responses have the function of auxiliary precurrent behavior, that is, behavior that increases the likelihood of reinforcement of the final response of a chain of responses, but it is not required by the programmed contingencies of reinforcement and occurs in situations that allow for the transference of stimulus functions. Auxiliary precurrent responses tend to decrease in duration and frequency as training increases (Oliveira-Castro et al., 1999; Oliveira-Castro et al., 2002).

Rule transmission is the combination of at least two interlocking behavioral patterns, that of conveying and that of adopting the rule. The reinforcement of conveying the rule, mediated or immediate, is the same as the reinforcement of adopting the rule: the modification of the behavioral repertoire of the one who adopts the rule, who now is capable of uttering the rule in appropriate problem situations and behaving according to its specification, that is, emitting responses topographically compatible to the rule. A rule has become part of the repertoire of an individual (or group of individuals) when he or she begins to use the utterance of said rule to solve the problems for which it is appropriate (Skinner, 1968, 1969). In other words, rule transmission is reinforced by changes in the repertoire of the recipient in the direction of applying the respective rule.

It is possible to speculate that, in the early days of human sociocultural evolution, the transmission of rules was like their creation, because, on the one hand, they were basically descriptions of the most common types of group-member behaviors (Skinner, 1953, p. 339). On the other hand, the contingency-specifying topographies of contemporary rules must be the result of the practice of transmitting them, which, over time, selected the types of verbal behavioral patterns most suitable for its learning. Such verbal behavioral patterns in turn facilitated the description of the group's customs, eventually giving rise, in combination with other factors, to "rule creation" as we know it today.

Thus, rule creation is a kind of behavioral pattern that must have emerged later in human sociocultural evolution. In fact, one can only properly speak of rule creation at the societal level when such behavior is reinforced by the behavioral change of a group 
of individuals. In the case of law, for example, it was only late in human sociocultural evolution that legislation began to be understood as the result of binding decisions by a legislator, that is, a politically legitimatized individual or group of individuals. For many centuries, the codification of laws was fundamentally a means of transmitting, as faithfully as possible, preexisting legal rules, not creating them (Luhmann, 1985). That said, we can define rule creation as the behavior of uttering rules reinforced by the behavior of transmitting and applying such rules by the individuals in society.

Table 1 succinctly presents the proposed typology of rule uttering and compares it to that presented by Baum $(2000,2005)$. It should be noted that the present proposal advances a tiered definition of rule uttering based on the concept of rule application. Rule transmission is reinforced by producing rule application, whereas rule creation is reinforced by producing rule transmission and rule application. Having presented our alternative typology of rule-uttering behaviors, the next issue to be solved is: how does the mechanism of rule selection work? As we will argue below, the answer is that rules are selected by means of the functional specialization of social systems and the concomitant emergence of rules for selecting rules: in short, second-degree rules.

\section{The Mechanism of Rule Selection}

Strictly speaking, the mechanism of rule selection is operant selection (Skinner, 1969; Van Parijs, 1981; Baum, 2000, 2005). In fact, the individual who did not solve the problem tends to abandon the rule used in trying to solve it. The instructor who transmits rules that are no longer required by the receivers will probably stop transmitting them and exchange them for more accepted ones. In turn, the rule creator is directly or indirectly an agent of social control, that is, someone whose behavior of uttering new rules, or of creating new, more effective formulations of preexisting rules, is reinforced by the behavior of others

Table 1. Comparison of rule-uttering typologies

\begin{tabular}{|c|c|c|}
\hline Author & Type of rule utterance & Main characteristics \\
\hline \multirow[t]{3}{*}{ Baum } & Rule-following & $\begin{array}{l}\text { Correlation between presentations of a contingency-specifying } \\
\text { verbal stimulus and emissions of a response compatible with } \\
\text { the topography related to the stimulus }\end{array}$ \\
\hline & Rule-giving & $\begin{array}{l}\text { Verbal behaviour under control of verbal discriminative stimuli } \\
\text { functionally equivalent to one another, motivated by "altruistic" } \\
\text { consequences }\end{array}$ \\
\hline & Rule-making & $\begin{array}{l}\text { Verbal behavior under discriminative control of regularities in } \\
\text { the natural environment, that precedes rule-giving, usually } \\
\text { associated to fitness }\end{array}$ \\
\hline \multirow[t]{3}{*}{ Present proposal } & Rule-application & $\begin{array}{l}\text { In the context of problem-situations, precurrent behavior of } \\
\text { uttering rules, that increase the likelihood of final responses } \\
\text { that might solve the problem }\end{array}$ \\
\hline & Rule-transmission & $\begin{array}{l}\text { Social behavioral pattern of uttering a rule, reinforced by } \\
\text { changes in the repertoire of the addressee in the direction } \\
\text { of applying the rule in appropriate problem-situations }\end{array}$ \\
\hline & Rule-creation & $\begin{array}{l}\text { Social behavioral pattern of uttering rules reinforced by the } \\
\text { behavior of transmitting and applying such rules by the } \\
\text { individuals of a given society }\end{array}$ \\
\hline
\end{tabular}


transmitting or applying those rules, or, for that matter, any reinforcing behavior of individuals for whom a change in the repertoire of these rules' final recipients is reinforcing (Skinner, 1953, 1968). It is important to emphasize that the distinction among rule application, rule transmission, and rule creation does not relate to fixed social roles. Indeed, the behavioral repertoires of, for example, modern scientists, economists, and jurists include behavioral patterns pertaining to the three modalities of rule uttering, which are selected by different contingencies experienced by these practitioners depending on the context.

If we consider the necessary interconnection among the three types of rule uttering and the ensuing functional similarity of the corresponding problems, we can conclude that, taken together, the recurrence of the behaviors of creating, transmitting, and applying rules leads to the formation of rule-selection cycles at the supra-individual level (Paterson \& Teubner, 1998; Teubner, 1993). In other words, the recurrent and interconnected cycles of functionally related problems (e.g., legal, scientific-technological, economic, or political) in which the three types of rule uttering occur, being nothing more than operant behavioral patterns, have their frequency of occurrence explained by the respective ensuing consequences for the individuals. Nevertheless, the combined effect of this selection at the individual level make up an emergent effect at a supra-individual level, especially because, as extensively argued throughout this article, rules are reinforced by the alteration of the behavioral repertoire of their recipients.

Indeed, starting with the premise that "it is always an individual who behaves," it is plausible to assume that Skinner is referring to something like these supra-individual rule-selection cycles when he speaks of effects for the group as the determining factor for sociocultural selection (Skinner, 1969, 1981, 1988). It is, in fact, a different level from those in which both operant selection and natural selection operate, however, without involving any acting collective entity, any form of sociocultural evolutionary progress, nor a subordination of sociocultural selection to the promotion of the fitness of individuals who create, transmit, or apply the rules of a given culture.

It is well-known that operant selection requires that the corresponding consequence be contingent to behavior. However, in the case of sociocultural contingencies, a major problem is that the great majority of them are the nondirect acting kind (Malott, 1989, 2009). How can such contingencies govern rule-uttering behavior, particularly considering that it is not just the sum of isolated individual behaviors but the integration of distinct behavioral patterns (creation, transmission, and application) present in the behavioral repertoire of literally billions of people, such as is the case with the economic system? The answer lies in the functional specialization of social systems, which Skinner calls controlling agencies (Skinner, 1953), for which the emergence of generalized social reinforcers, such as money (economy), sanctions (law), votes (government), empirical data (science), and grades (education), is paramount (Luhmann, 1990).

The case of the economic system is perhaps the most illustrative. As Skinner puts it, money is the generalized social reinforcer of the economy (Skinner, 1953). In fact, the rules created by economists, through concepts such as opportunity cost, sunk costs, and economic profit (Friedman, 1986), do not directly concern the reinforcement obtained with the use of the money won or saved by the application of such rules, but to the presupposed reinforcement ensuing from obtaining or maintaining money itself as generalized social reinforcer. In other words, in the jargon of economists, the preferences of individuals are exogenous to the models of economic analysis and, in our terminology, to the rules of economic (efficient) behavior derived from such models. 
We can then characterize the economic system as a set of interlocked behavioral patterns aimed at (reinforced by) obtaining the generalized social reinforcer money.

The same analysis applies to the law: the sanctions enforced by the authorities for noncompliance with legal rules do not depend on their personal judgment about the sanctioned behavior, but on the legality or illegality of such behavior (Hart, 1961; Luhmann, 1985, 2004). Moreover, the classification of a conduct as legal or illegal does not necessarily depend on its intrinsically beneficial or evil nature, but on a decision made by the individual or group of individuals politically legitimized to do so (Kelsen, 1967). Thus, in modern legal systems the difference between legal and illegal behaviors is defined by the fact that there are sanctions designed to be applied to the latter but not to the former. Therefore, the law can be defined as a set of interlocked behavioral patterns aimed at (reinforced by) the punishment of certain behaviors considered socially harmful (de Aguiar, 2017).

For economic, legal, or any functionally specialized social system to work, the creation, transmission, and application - in a word, the selection-of rules for the respective appropriate behaviors is crucial. Therefore, in parallel to the historic development of such functionally specialized social systems, there emerged the so-called rules for rule selection, or second-degree rules (Skinner, 1953, 1969), whose paradigmatic example are the rules of logic and scientific methodology aimed at the selection of scientific rules (Skinner, 1957). Like any rule, the probability of occurrence of second-degree rules depends on their ability to change the behavior of an individual or group of individuals. In their case, it is about rule-creation behavior, in order to create more effective rules or to propose new, more effective formulations of preexisting rules. From the emergence of functionally specialized social systems and their respective second-degree rules, a hypercycle (Paterson \& Teubner, 1998; Teubner, 1993) of recurring cycles of creation, transmission, and application of rules for each system is formed on a societal level (i.e., the supra-individual level) without it being necessary to presuppose any other basic behavioral process besides the operant reinforcement.

\section{Conclusion}

This article presented the thesis of rules as basic units of sociocultural selection, based on a critical analysis of the main ideas on the subject proposed by Skinner and Baum. The core of our thesis is a new functional concept of rules, which are defined as special kinds of verbal behavioral patterns whose probability of occurrence depend on their ability to change the frequency of occurrence of a certain type of behavior in the repertoire of an individual or group of individuals.

From this definition, we tried to show, first, why Baum's thesis that cultural practices - defined as behavioral patterns characteristic of a particular social groupare the basic units of sociocultural selection cannot be sustained. Our main argument was that the concept of cultural practice only denotes a fact, but does not explain it causally, unlike the functional concept of rules, which allows us to equate and solve the main problem of the radical behavioralist theory of sociocultural selection, that is, how to conciliate the operant selection basic mechanism with the fact that sociocultural contingencies are of the type that "does not act directly." 
Finally, we proposed a rule-selection mechanism. First, the typology of the behavioral patterns of rule uttering proposed by Baum was critically discussed, and another typology was advanced, which is composed of three categories: rule creation, rule transmission, and rule application. Having discussed the main characteristics of each type of rule-uttering behavior, it was postulated that the mechanism of rule selection is the functional specialization of social systems accompanied by the corresponding emergence of second-degree rules. These second-degree rules have the defining characteristic of having their frequency of occurrence dependent on the ability to select the most effective rule-creation behavior in each functionally specialized social system.

Acknowledgments Julio C. Aguiar (\#193.001.634/2017) and Jorge M. Oliveira-Castro (\#308859/2011-1, \#310528/2015-1) received research grants from FAPDF (Federal District Research Foundation, Brazil) and CNPq (National Council for Scientific and Technological Development, Brazil). The authors gratefully acknowledge these sources of financial support.

\section{Compliance with Ethical Standards}

Conflict of Interest Julio C. Aguiar, Jorge M. Oliveira-Castro, and Leandro Gobbo declare that they have no conflict of interest.

Ethical Standards The research protocols described in this article were determined to be exempt from review by the committee.

\section{References}

Acemoglu, D., \& Robinson, J. A. (2012). Why nations fail: The origins of power, prosperity, and poverty. New York, NY: Crown Business.

Alexander, R. D. (1987). The biology of moral systems. New York, NY: de Gruyter.

Baum, W. M. (1995). Rules, culture, and fitness. The Behavior Analyst, 18(1), 1-21. https://doi.org/10.1007 /bf03392688.

Baum, W. M. (2000). Being concrete about culture and cultural evolution. In N. Thompson \& F. Tonneau (Eds.), Perspectives in ethology: Evolution, culture, and behavior. New York, NY: Kluwer Academic/ Plenum.

Baum, W. M. (2005). Understanding behaviorism: Behavior, culture and evolution (2nd ed.). Oxford, UK: Blackwell.

Betzig, L. L. (1986). Despotism and differential reproduction: A Darwinian view of history. London: Aldine.

Blakely, E., \& Schlinger, H. (1987). Rules: Function-altering contingency-specifying stimuli. The Behavior Analyst, 10(2), 183-187. https://doi.org/10.1007/bf03392428.

Boudon, R. (1977). Effects pervers et ordre social. Paris, France: Presses Universitaires de France.

Campbell, D. T. (1974). Evolutionary epistemology. In P. A. Schilpp (Ed.), The philosophy of Karl R. Popper (pp. 413-463). La Salle, IL: Open Court.

Catania, A. C. (1998). Learning (4th ed.). Upper Saddle River, NJ: Prentice Hall.

Corning, P. A. (2014). Evolution "on purpose": How behavior has shaped the evolutionary process. Biological Journal of Linnean Society, 114, 242-260. https://doi.org/10.1111/bij.12061.

Corsi, G., Esposito, E., \& Baraldi, C. (1996). Glosario sobre la teoría Social de Niklas Luhmann. (M. R. Pérez \& C. Villalobos, under the coordination of J. T. Nafarrate, trans.). Mexico City, Mexico: Universidad Iberoamericana Universidad Iberoamericana, Instituto Tecnológico y de Estudios Superiores de Occidente (ITESO), and Editorial Anthropos.

de Aguiar, J. C. (2017). Teoria analítico-comportamental do Direito. Porto Alegre, Brazil: Núria Fabris.

Eggan, F. (1963). Cultural drift and social chance. Current Anthropology, 4(4), 347-355. https://doi. org/10.1086/200393.

Friedman, D. D. (1986). Price theory: An intermediate text. Cincinnati, OH: Southwestern. 
Glenn, S. S. (1988). Contingencies and metacontingencies: Toward a synthesis of behavior analysis and cultural materialism. The Behavior Analyst, 27, 133-151. https://doi.org/10.1007/bf03392470.

Glenn, S. S. (2003). Operant contingencies and the origin of cultures. In K. A. Lattal \& P. N. Chase (Eds.), Behavior theory and philosophy (pp. 223-242). New York, NY: Kluwer Academic/Plenum.

Glenn, S. S. (2004). Individual behavior, culture, and social change. The Behavior Analyst, 27, $133-151$. https://doi.org/10.1007/bf03393175.

Gosselin, A. (1998). La logique des effects pervers. Paris, France: Presses Universitaires de France.

Hardin, G. (1968). The tragedy of the commons. Science, 162(3859), 1243-1248. https://doi.org/10.1126 /science.162.3859.1243.

Hart, H. L. A. (1961). The concept of law. Oxford, UK: Oxford University Press.

Kelsen, H. (1967). Pure theory of law. (M. Knight, trans.). Berkeley, CA: University of California Press.

Kunkel, J. H. (1975). Behavior, social problems, and change. Englewood Cliffs, NJ: Prentice Hall.

Kunkel, J. H. (1986). The Vicos Project: A cross-cultural test of psychological propositions. The Psychological Record, 36(4), 451-466. https://doi.org/10.1007/bf03394965.

Kunkel, J. H. (1991). Apathy and irresponsibility in social systems. In P. A. Lamal (Ed.), Behavioral analysis of societies and cultural practices (pp. 219-240). (Series in Health Psychology and Behavioral Medicine). Washington, DC: Hemisphere Publishing.

Luhmann, N. (1985). A sociological theory of law. (E. King \& M. Albrow, trans.). London, UK: Routledge \& Kegan Paul.

Luhmann, N. (1990). Essays on self-reference. New York, NY: Columbia University Press.

Luhmann, N. (2004). Law as a social system. (K. A. Ziegart., trans.). Oxford, UK: Oxford University Press.

Malott, R. W. (1989). The achievement of evasive goals: Control by rules describing contingencies that are not direct acting. In S. C. Hayes (Ed.), Rule-governed behavior: cognition, contingencies \& instructional control (pp. 269-322). Reno, NV: Context Press.

Malott, R. W. (2009). Principles of behavior (6th ed.). Upper Saddle River, NJ: Pearson Education.

Mezan, R. (1982). Freud: A trama dos conceitos. São Paulo, Brazil: Perspectiva.

Michael, J. (1982). Distinguishing between discriminative and motivating functions of stimuli. Journal of the Experimental Analysis of Behavior, 37(1), 149-155. https://doi.org/10.1901/jeab.1982.37-149.

Moeller, H.-G. (2006). Luhmann explained: From souls to systems. La Salle, IL: Open Court.

Moore, J. (2008). Conceptual foundations of radical behaviorism. In Cornwall-on-Hudson. NY: Sloan.

North, D. C., Wallis, J. J., \& Weingast, B. R. (2009). Violence and social orders: A conceptual framework for interpreting recorded human history. New York, NY: Cambridge University Press.

Oliveira-Castro, J. M., Coelho, D. S., \& Oliveira-Castro, G. A. (1999). Decrease of precurrent behavior as training increases: Effects of task complexity. The Psychological Record, 49(2), 299-325. https://doi. org/10.1007/bf03395322.

Oliveira-Castro, J. M., Faria, J., Dias, M., \& Coelho, D. S. (2002). Effects of task complexity on learning to skip steps: An operant analysis. Behavioural Processes, 59(2), 101-120. https://doi.org/10.1016/s03766357(02)00087-6.

Paterson, J., \& Teubner, G. (1998). Changing maps: Empirical legal autopoiesis. Social \& Legal Studies, 7 , 451-486. https://doi.org/10.1177/096466399800700401.

Rachlin, H. (2000). The science of self-control. Cambridge, MA: Harvard University Press.

Schelling, T. C. (1978). Micromotives and macrobehavior. New York, NY: Norton.

Schlinger, H. D. (1993). Separating discriminative and function-altering effects of verbal stimuli. The Behavior Analyst, 16(1), 9-23. https://doi.org/10.1007/bf03392605.

Simmel, G. (1902). The number of members as determining the form of the group: I. American Journal of Sociology, 8(1), 1-46. https://doi.org/10.1086/211115.

Skinner, B. F. (1953). Science and human behavior. New York, NY: Free Press.

Skinner, B. F. (1957). Verbal behavior. New York, NY: Appleton-Century-Crofts.

Skinner, B. F. (1968). The technology of teaching. New York, NY: Appleton-Century-Crofts.

Skinner, B. F. (1969). Contingencies of reinforcement: A theoretical analysis. New York, NY: AppletonCentury-Crofts.

Skinner, B. F. (1971). Beyond freedom and dignity. New York, NY: Knopf.

Skinner, B. F. (1976). About behaviorism. New York, NY: Vintage Books.

Skinner, B. F. (1981). Selection by consequences. Science, 213(4507), 501-504. https://doi.org/10.1126 /science. 7244649 .

Skinner, B. F. (1988). Canonical papers of B. F. Skinner. In A. C. Catania \& S. Harnad (Eds.), The selection of behavior: The operant behaviorism of B. F. Skinner: Comments and consequences. New York, NY: Cambridge University Press. 
Skinner, B. F. (1989). The behavior of the listener. In S. C. Hayes (Ed.), Rule-governed behavior: Cognition, contingencies \& instructional control (pp. 85-96). Reno, NV: Context Press.

Teubner, G. (1993). Law as an autopoietic system. Oxford, UK: Blackwell.

Van Parijs, P. (1981). Evolutionary explanations in the social sciences. London, UK: Tavistock.

Zettle, R. D. (1990). Rule-governed behavior: A radical behavioral answer to the cognitive challenge. The Psychological Record, 40(1), 41-49. https://doi.org/10.1007/bf03399570.

Publisher's Note Springer Nature remains neutral with regard to jurisdictional claims in published maps and institutional affiliations. 